\title{
Meta
}

Journal des traducteurs

Translators' Journal

\section{La technologie langagière au Secrétariat d'État du Canada : une réalité quotidienne}

\section{Klaire Tremblay}

Volume 37, numéro 4, décembre 1992

Études et recherches en traductique / Studies and Researches in Machine Translation

URI : https://id.erudit.org/iderudit/004506ar

DOI : https://doi.org/10.7202/004506ar

Aller au sommaire du numéro

Éditeur(s)

Les Presses de l'Université de Montréal

ISSN

0026-0452 (imprimé)

1492-1421 (numérique)

Découvrir la revue

Citer cet article

Tremblay, K. (1992). La technologie langagière au Secrétariat d'État du Canada : une réalité quotidienne. Meta, 37(4), 761-769. https://doi.org/10.7202/004506ar

\section{Résumé de l'article}

Depuis le début des années 70, le Canada montre un vif intérêt pour tout ce qui touche le traitement électronique du langage. Le projet TAUM MÉTÉO et la banque de terminologie TERMWM en sont d'excellents exemples. Le Secrétariat d'État se livre maintenant à l'essai, en milieu opérationnel, de deux outils de traduction haute technologie, soit du logiciel de TAO LOGOS et du poste de travail du traducteur (PTT). Les expériences menées jusqu'à ce jour ont donné lieu à diverses évaluations techniques ainsi qu'à des études linguistiques et ergonomiques, notamment à l'établissement d'une typologie des textes pouvant se prêter au traitement machine. Le Secrétariat d'État travaille dorénavant en étroite collaboration avec la société Logos et avec le centre de recherche responsable de la conception du PTT afin d'améliorer les prototypes à la lumière des besoins et des recommandations des traducteurs. Outre les deux grands projets-pilotes susmentionnés, le Secrétariat d'État vient également de doter l'ensemble de ses 800 traducteurs de micro-ordinateurs haut de gamme et il procède actuellement à la création d'une trousse d'ouvrages électroniques. De plus, il collabore à la mise au point d'une station de travail pour terminologue appelée LATTER (L'ATelier du TERminologue), dont le premier prototype sera mis à l'essai à l'été 1992. Toutes ces entreprises tendent vers un but à long terme, soit l'intégration en un seul et même système des meilleures aides à la traduction disponibles sur le marché. C'est ainsi que le traducteur de demain pourra, de son poste de travail, communiquer directement avec son client, consulter toutes les sources de documentation et les bases de données terminologiques électroniques nécessaires, confier la traduction de certains passages à un logiciel de traduction et, le cas échéant, procéder à l'éditique de son texte. Les possibilités sont immenses dans le domaine du traitement des langues naturelles, et le Secrétariat d'État du Canada déploie des efforts soutenus afin de demeurer à la tête du peloton.
Ce document est protégé par la loi sur le droit d'auteur. L'utilisation des services d'Érudit (y compris la reproduction) est assujettie à sa politique d'utilisation que vous pouvez consulter en ligne.

https://apropos.erudit.org/fr/usagers/politique-dutilisation/ 


\title{
LA TECHNOLOGIE LANGAGIÈRE AU SECRÉTARIAT D'ÉTAT DU CANADA: UNE RÉALITÉ QUOTIDIENNE
}

KLAIRE TREMBLAY

Secrétariat d'État du Canada, Ottawa, Canada

\begin{abstract}
Résumé
Depuis le début des années 70 , le Canada montre un vif intérêt pour tout ce qui touche le traitement électronique du langage. Le projet TAUM MÉTÉO et la banque de terminologie TERMIUM en sont d'excellents exemples. Le Secrétariat d'État se livre maintenant à l'essai, en milieu opérationnel, de deux outils de traduction haute technologie, soit du logiciel de TAO LOGOS et du poste de travail du traducteur (PTT). Les expériences menées jusqu'à ce jour ont donné lieu à diverses évaluations techniques ainsi qu'à des études linguistiques et ergonomiques, notamment à l'établissement d'une typologie des textes pouvant se prêter au traitement machine. Le Secrétariat d'État travaille dorénavant en étroite collaboration avec la société Logos et avec le centre de recherche responsable de la conception du PTT afin d'améliorer les prototypes à la lumière des besoins et des recommandations des traducteurs.

Outre les deux grands projets-pilotes susmentionnés, le Secrétariat d'État vient également de doter l'ensemble de ses 800 traducteurs de micro-ordinateurs haut de gamme et il procède actuellement à la création d'une trousse d'ouvrages électroniques. De plus, il collabore à la mise au point d'une station de travail pour terminologue appelée LATTER (L'ATelier du TERminologue), dont le premier prototype sera mis à l'essai à l'été 1992. Toutes ces entreprises tendent vers un but à long terme, soit l'intégration en un seul et même système des meilleures aides à la traduction disponibles sur le marché. C'est ainsi que le traducteur de demain pourra, de son poste de travail, communiquer directement avec son client, consulter toutes les sources de documentation et les bases de données terminologiques électroniques nécessaires, confier la traduction de certains passages à un logiciel de traduction et, le cas échéant, procéder à l'éditique de son texte. Les possibilités sont immenses dans le domaine du traitement des langues naturelles, et le Secrétariat d'État du Canada déploie des efforts soutenus afin de demeurer à la tête du peloton.
\end{abstract}

\begin{abstract}
Since the early 1970s Canada has been keenly interested in all aspects of electronic language processing. The TAUM MÉTÉO project and the TERMIUM terminology bank are excellent examples of this interest. The Secretary of State Department has now begun operational testing of two high-technology translation tools, the LOGOS CAT software and the translator's work station (TWS). The experiments so far have given rise to various technical evaluations and to linguistic and ergonomic studies, particularly the establishment of a typology of texts suitable for processing by computer: From now on the Secretary of State Department will be working closely with the Logos firm and the research centre that designed the TWS to improve the prototypes in the light of needs and the translators' recommendations.

Besides the two major pilot projects described above, the Secretary of State Department has given all its 800 translators sophisticated microcomputers and is now creating an "electronic desk set". It is also co-operating in the development of a terminologist's work station called LATTER (L'ATelier du TERminologue); testing of the first prototype will begin in the summer of 1992. The long-term goal of all these projects is to integrate into a single system the best translation aids available on the market. Thus, without leaving his or
\end{abstract}


her work station, the translator of tomorrow will be able to communicate directly with the client, consult all the necessary documentation sources and terminology data bases, assign the translation of certain passages to a computer translation program, and process the text using a desktop publication program if necessary. The possibilities for processing natural languages are immense, and the Secretary of State Department is making a sustained effort to remain in the forefront of the technology.

En tant que fournisseur officiel de services linguistiques, le Bureau de la traduction s'intéresse depuis très longtemps à la technologie langagière et, plus particulièrement, à la traduction assistée par ordinateur (TAO). Les impératifs d'une demande sans cesse croissante (plus de 297 millions de mots annuellement) l'ont amené graduellement à participer aux activités de recherche et développement dans des domaines que l'on regroupe sous les vocables de traductique, de bureautique et d'éditique.

Les premiers travaux débutent au milieu des années 60 , au moment de la création du Centre d'études pour le traitement automatique de données linguistiques (CETADOL) à l'Université de Montréal. Les recherches de ce centre portent alors notamment sur les nouvelles théories linguistiques (par exemple, la grammaire générative — transformationnelle), sur la théorie des automates et des grammaires formelles et sur l'expérimentation des principaux algorithmes d'analyse syntaxique existants. Au début des années 70 , le CETADOL précise son orientation et devient le Groupe de recherche en traduction automatique de l'Université de Montréal (TAUM). Les chercheurs se concentrent dès lors sur la conception et la mise au point d'un premier prototype de système de traduction automatique de deuxième génération. En 1973, le Bureau de la traduction prend le relais et s'associe au Groupe TAUM. Trois ans plus tard, il reçoit le système TAUM-MÉTÉO, qu'il installe au Centre météorologique canadien. Le Groupe TAUM se lance également dans la conception d'un système en vue de la traduction automatique de manuels d'entretien de l'aéronef AURORA (CF-140) du ministère de la Défense nationale. Le projet, appelé TAUM-AVIATION, est abandonné en raison des piètres résultats d'une étude de faisabilité effectuée par le Secrétariat d'État. De nos jours, TAUM-MÉTÉO est le seul système du genre qui soit opérationnel. Désormais connu sous le nom MÉTÉO, il traduit vingt-quatre heures par jour, de l'anglais au français et vice versa, sans intervention humaine importante, au delà de 17 millions de mots par année. Sur les quelque 175 bulletins météorologiques traduits au cours d'une période de vingt-quatre heures, $80 \%$ le sont automatiquement et $20 \%$ manuellement.

Dans la deuxième partie des années 70 , le Bureau de la traduction entreprend également un autre projet d'envergure qui, comme TAUM-MÉTÉO, deviendra l'un des plus grands succès en traductique. Il s'agit bien sûr de la Banque de terminologie TERMIUM. Le fichier de base est élaboré en 1975, à partir des quelque 150000 fiches de la banque de terminologie informatisée de l'Université de Montréal et des centaines de milliers de fiches accumulées au fil des ans par les traducteurs du Bureau. Deux ans plus tard, le système est transféré sur l'ordinateur du ministère de l'Énergie, des Mines et des Ressources, à Ottawa. Le Bureau met par la suite sur pied son propre système à partir du progiciel BASIS et d'une structure de menus facilitant l'accès à l'information. Outre qu'il fonctionne en ligne, TERMIUM est accessible sur DOC (CD-ROM) depuis 1990. La Banque offre maintenant aux usagers deux grandes bases de données, soit la base de données linguistiques et la base de données documentaires. D'autres fiches traitent des problèmes de traduction (phraséologie, proverbes et dictons) ou des problèmes de langue (sémantique, morphologie, stylistique, etc.). Enfin, il existe aussi des fiches multilingues comportant des termes en langues étrangères associées ou non à l'une des deux langues officielles du Canada, soit le français et l'anglais. La dernière version du DOC compte près d'un million de fiches bilingues pour environ 3 millions de termes dans tous les domaines de la connaissance. 
Dans la foulée technologique du début des années 80 , le Bureau, fort de son expérience, décide de mettre à l'essai deux logiciels de traduction, à savoir Microcat de Weidner et Alps. Les deux expériences, d'une durée d'environ six mois chacune, se soldent par un échec. Malgré les efforts soutenus d'enrichissement des dictionnaires consentis par les traducteurs, la qualité linguistique du produit brut demeure inacceptable. Les outils se révèlent également coûteux. Mais le Bureau ne s'avoue pas vaincu. La mise sur le marché de systèmes informatiques plus performants et l'avènement de l'intelligence artificielle l'incitent à tenter une nouvelle expérience de TAO. Il décide alors que le nouveau projet pilote se déroulera sur une période prolongée, à savoir cinq ans, dans un environnement contrôlé et bien délimité.

\section{PROJET LOGOS}

Le choix du Bureau s'arrête sur le logiciel Logos, qui, bien qu'il soit particulièrement adapté à la traduction scientifique et technique, peut être utilisé dans divers champs d'application. Dans une première phase, le Bureau crée trois bancs d'essai appelés sites TAO. Les services de traduction devant accueillir un site sont choisis en fonction du volume de la demande et de la technicité des textes. Un calendrier d'implantation et de mise en ouvre est établi de concert avec la société Logos Canada. Le premier site TAO voit le jour à la Section informatique, à Montréal, en 1987. L'année suivante, la Section de la Défense nationale et la Section technique, situées toutes deux à Ottawa, accueillent respectivement le deuxième et le troisième. Au total, 17 traducteurs et personnes de soutien travaillent alors à plein temps à la TAO. Les traducteurs ont essentiellement pour rôle d'évaluer les valeurs ergonomiques et linguistiques de l'outil de travail et d'en déterminer l'utilité en regard des besoins et des exigences du Bureau de la traduction.

Compte tenu de l'ampleur que prennent les diverses initiatives liées à la TAO, le Bureau juge bon de créer le Secrétariat général TAO au début de 1989. Ce comité représente les divers secteurs intéressés et a pour mandat de planifier, d'organiser et d'administrer les activités de TAO sur les plans linguistique, informatique et technologique. Il lui incombe notamment d'établir et de mettre en application des normes linguistiques, de coordonner les travaux de recherche et de développement dans le domaine et de fournir aux sites TAO l'aide technique dont ils ont besoin. Le Secrétariat général rédige chaque année un Bilan TAO dans lequel il analyse les résultats obtenus pendant l'année et les compare à ceux des années précédentes. En mai 1989, le Bureau crée, en collaboration avec les sociétés Wang Canada et Logos Canada, un Centre d'expertise chargé de mener à bien les recherches et les essais linguistiques et techniques nécessaires. Toujours en 1989, il procède à l'aménagement d'une salle de démonstration et de formation TAO à proximité du Centre d'expertise.

Pendant les deux premières années, les traducteurs font montre d'un grand enthousiasme, et les progrès sont considérables. Compte tenu des résultats encourageants et à la demande de ministères clients, le Bureau décide d'intensifier ses efforts en créant trois nouveaux sites. C'est ainsi qu'en 1989 et en 1990 , les services de traduction de Transports Canada, du ministère de l'Agriculture et du Quartier général de la Force mobile joignent les rangs de la TAO. À l'heure actuelle, cinq de ces six sites poursuivent l'essai, le ministère de l'Agriculture ayant été obligé de se retirer pour des raisons d'ordre opérationnel.

Le Bureau profite de l'implantation des trois nouveaux sites pour améliorer le matériel des équipes TAO et pour doter celles-ci d'autres outils électroniques utiles tels que vérificateurs orthographiques et logiciels de conjugaison. Chaque traducteur (appelé taoïste dans le jargon du métier) dispose dorénavant d'un micro-ordinateur haut de gamme plutôt que d'un simple terminal Wang et d'une imprimante laser. Le logiciel 
WordPerfect a remplacé le traitement de texte WP de Wang, devenu désuet. De plus, pour faciliter le travail du personnel de soutien, les sites ont maintenant à leur disposition d'autres périphériques indispensables comme le lecteur de caractères optiques Kurzweil pour la saisie des textes sur papier et le système de conversion des fichiers de traitement de textes Keyword. Des modems facilitent également la transmission de données et le dépannage à distance.

Pendant les trois premières années du projet, le Bureau met au point une procédure d'implantation TAO touchant tous les aspects pratiques, à savoir les critères de sélection d'un site, les besoins en ressources de toutes sortes, la formation des participants et les mécanismes de collecte de données, pour n'en nommer que quelques-uns. Toutefois, il ne dispose alors d'aucun moyen d'évaluer et de mesurer scientifiquement la performance du système Logos, ou de tout autre logiciel de traduction. C'est pourquoi, en 1990, il met au point une méthodologie d'évaluation (Bourbeau 1990) qui lui permettra de mesurer le degré d'utilité du produit et de déterminer toutes les conditions à remplir pour en justifier l'utilisation. La méthodologie fournit notamment un inventaire et une classification des diverses méthodes d'évaluation des systèmes TAO. De plus, elle expose les éléments fondamentaux d'une évaluation globale, soit les critères linguistiques, les échelles de cotation, les grilles d'évaluation, les typologies de classification et les méthodes d'application.

À la même époque, le Secrétariat général TAO procède à l'évaluation linguistique de la version 6.0 de Logos, nouvellement installée dans les sites (Secrétariat d'État du Canada 1990). Cette version intègre des changements importants, notamment une restructuration du dictionnaire. La méthodologie Bourbeau n'étant pas encore tout à fait au point, l'évaluation consiste simplement en une analyse comparée de la traduction brute d'un corpus de quelque 400 phrases à l'aide de la version 6.0 et de la version précédente. Trois critères figurant au rapport ALPAC sont retenus, soit la fidélité (qualité du transfert), la qualité linguistique (respect des règles usuelles de grammaire) et la postédition (la complexité des manipulations requises pour que la phrase atteigne la qualité voulue). Une grille de cotation à sept niveaux est établie pour chacun de ces trois facteurs. Toutefois, la méthode d'évaluation utilisée n'est pas suffisamment scientifique pour effectuer une analyse poussée.

Le Bureau met donc au point un corpus d'évaluation linguistique de systèmes de TAO (Bourbeau 1991), qui constitue en fait le deuxième volet de la méthodologie d'évaluation des systèmes mentionnée plus haut. Le corpus se divise essentiellement en deux parties, soit un corpus témoin dont chaque phrase isole un phénomène linguistique particulier et un corpus de contrôle du corpus témoin. Le fabricant peut avoir accès au corpus témoin, tandis que le corpus de contrôle constitue en quelque sorte la «boîte noire» de l'évaluateur. Ce deuxième corpus permet principalement de valider les résultats obtenus à l'aide du corpus témoin. Le nouveau corpus sert dorénavant à l'évaluation linguistique des versions successives de Logos, ainsi que des autres logiciels de traduction qui sont soumis au Bureau par les divers fabricants.

Depuis le lancement du projet pilote en 1987, les sites présentent des demandes ponctuelles directement au fournisseur. II s'agit en fait de recommandations écrites visant soit l'ajout de termes complexes au dictionnaire, soit la modification du programme permettant la rédaction de règles sémantiques, soit l'amélioration du transfert de certains types de phrases. Par suite de la multiplication des sites, le Bureau s'aperçoit que le nombre de demandes dépasse le millier et que certaines d'entre elles présentent des discordances. Il met donc sur pied le Sous-comité de coordination des demandes à Logos, dont le mandat se passe d'explications. Pour se doter des outils nécessaires, le Souscomité crée une typologie des principaux problèmes du logiciel et une typologie cumulative des demandes. Grâce au répertoire établi, il se rend rapidement compte que le gros 
des demandes a trait au dictionnaire. Toutefois, comme les problèmes d'analyse influent directement sur la qualité de la traduction brute, il décide de travailler désormais étroitement avec le centre de recherche et développement de Logos aux États-Unis. Grâce à cette collaboration, le Bureau est en mesure de mieux définir ses besoins auprès des concepteurs et d'intervenir même à l'étape d'élaboration de certains programmes.

On ne peut cependant entreprendre un projet de TAO de grande envergure sans se heurter à des difficultés majeures. Malgré les ressources financières et humaines investies et en dépit des nombreuses initiatives amorcées, des problèmes d'ordre opérationnel subsistent. Mentionnons d'abord le long travail d'enrichissement qu'exigent le dictionnaire et la base de données sémantiques. Malgré les efforts des traducteurs, le produit brut requiert souvent une postédition soutenue. Deuxièmement, les services informatiques doivent fournir un appui technique régulier au logiciel Logos et au mini-ordinateur Wang qui lui sert de plate-forme. Vu la complexité du matériel, il y a souvent des pannes, que les traducteurs ne sont pas en mesure de régler. À vrai dire, les interruptions font partie du quotidien du taoïste.

Ensuite, l'approvisionnement en textes susceptibles de se prêter au traitement machine (que les initiés appellent textes taoïsables) fait problème encore aujourd'hui. En effet, comme bon nombre des documents volumineux ne répondent pas nécessairement aux critères des textes taoïsables, les équipes TAO sont souvent obligées de traduire des textes courts, dans des domaines qui conviennent plus ou moins au système. En conséquence, le dictionnaire doit constamment être mis à jour. Par ailleurs, de nombreux documents ne se trouvent pas sur support ordinolingue. Il faut alors avoir recours à un lecteur optique, aux résultats souvent imprévisibles. Lorsque les textes sont fournis sur disquette, il n'est pas rare que le traitement de texte utilisé soit incompatible avec Logos. Il faut alors procéder à une conversion, ce qui entraîne normalement d'interminables heures de formatage. Outre ces opérations purement techniques, les traducteurs effectuent une pré-édition importante afin que le texte de départ corresponde à un protocole de rédaction acceptable pour Logos (ajout de ponctuation et modification des phrases pour désambiguiiser le signifié). Toutes ces tâches deviennent à la longue fastidieuses pour le traducteur.

Outre les difficultés susmentionnées, inhérentes à tout projet de TAO, un autre facteur retient notre attention, soit la formation des traducteurs. Lors de l'implantation d'un nouveau site ou de roulement de personnel, la formation des traducteurs à l'utilisation du système de traduction èt des logiciels périphériques exige une période relativement longue qui se solde habituellement par une baisse de productivité. Les traducteurs disposent toutefois de tout le temps nécessaire pour se familiariser avec leur nouvel outil. De cette façon, ils sont en mesure d'acquérir une connaissance approfondie du système et d'exiger du fournisseur que le produit soit adapté à leurs besoins et non pas le contraire.

La performance d'un logiciel de traduction ne peut s'évaluer uniquement en fonction de la qualité du produit brut ou de la vitesse du traitement électronique. Il faut aussi tenir compte de la production, des investissements financiers, des qualités ergonomiques et, dans une certaine mesure, du temps consenti au projet. Dans cette optique, le Bureau se livre chaque année à une analyse exhaustive de la productivité et de la rentabilité afin de mesurer les progrès réalisés. Le rendement global des taoïstes est également comparé à celui des traducteurs de l'ensemble du Bureau.

Bien que le projet pilote en TAO ne porte que sur le logiciel Logos, le Bureau s'intéresse de près à tous les autres outils de traductique offerts sur le marché. Dans cette optique, il collabore étroitement avec Statistique Canada à un projet d'éditique et de traductique intitulé Docutech/Docutran. Cette nouvelle entreprise a pour but de doter les traducteurs des moyens électroniques nécessaires à la production de publications et de 
formulaires bilingues. Le système utilisé présente deux composantes, soit Systran pour la traduction automatique et GlobalView (de Xerox) pour la publication assistée par ordinateur, mieux connue sous le nom d'éditique. Si le système donne le rendement escompté, une implantation sur une plus grande échelle permettra à Statistique Canada de réaliser des économies considérables pour ce qui est des délais et des coûts de conception, de composition et de traduction.

\section{PROJET PTT (POSTE DE TRAVAIL DU TRADUCTEUR)}

Le Bureau s'est vite rendu compte que les automates de la traduction ne pourraient jamais à eux seuls répondre à une demande sans cesse changeante. D'une part, bon nombre de traducteurs demeurent réfractaires à l'idée de réviser un produit machine et, d'autre part, les clients demandent des services de plus en plus variés (reproduction graphique, mise en page, conception, transmission électronique, etc.). Le Bureau décide donc de tirer avantage de l'évolution rapide de la micro-informatique et des nouveaux outils électroniques qui apparaissent sur le marché. En 1988, il confie au Centre canadien de recherche sur l'informatisation du travail (CCRIT) le mandat de concevoir un poste de travail du traducteur (PTT) très perfectionné.

Le prototype devant être mis au point repose sur un concept général, soit l'exploitation de matériel et de logiciels courants permettant d'informatiser, ou d'accélérer, certaines fonctions liées à l'acte de traduction, ce dernier demeurant le fait du traducteur même. Toutes les fonctionnalités du poste doivent, évidemment, répondre aux besoins et aux attentes des futurs utilisateurs. Le PTT 1 fait son apparition en 1989.

L'enveloppe de l'outil comporte un micro-ordinateur AT 286 avec écran pleine page monochrome, une imprimante matricielle et une souris. Pour leur part, les aides informatisées regroupent le traitement de texte à fonctions augmentées WordPerfect, qui constitue en quelque sorte la base du poste. Les vérificateurs orthographiques français (Chandioux) et anglais (UK de WordPerfect) sont également inclus. Viennent ensuite se greffer le logiciel de comparaison de textes CompareRite, qui décèle les passages ajoutés, supprimés ou modifiés; l'outil de recherche documentaire Textsearch, qui relève, entre autres, les occurrences de différents termes; le logiciel de conjugaison SM VERBES, semblable au Bescherelle; le logiciel de gestion de données terminologiques Termex, qui permet notamment la création et la fusion de glossaires maison; l'outil de conversion de fichiers de traitement de texte Software Bridge; et les dictionnaires électroniques bilingues Harrap's et Robert \& Collins. Pour gérer efficacement et simultanément toutes ces applications en mémoire centrale, on fait appel à l'intégrateur DESQView.

Trois services de traduction sont choisis comme bancs d'essai (appelés sites PTT), à savoir les Sections Finances et Conseil du Trésor, Économique et Juridique et Communications. Les traducteurs, pour la plupart, sont des néophytes en informatique. Ils ont pour tâche non seulement de traduire en utilisant la technologie, mais aussi de vérifier la convivialité du prototype dans la réalité des opérations, de relever, s'il y a lieu, les lacunes des outils mis à leur disposition, et de formuler des recommandations quant à leurs besoins pour mieux accomplir leur travail. Pendant un an, les traducteurs mettent à l'essai le poste de travail en milieu opérationnel. Au cours de cette période, le Bureau tient une série de rencontres avec les utilisateurs afin de connaître l'utilité, l'efficacité et la convivialité de chacune des composantes du poste.

À l'issue de la première année d'essai, le CCRIT procède à l'évaluation de la convivialité du PTT (CCRIT 1991). L'étude comporte trois volets, soit les qualités ergonomiques de l'interface (facilité d'utilisation), la fonctionnalité (pertinence des applications dans le contexte du travail du traducteur) et la relation avec le contexte socioorganisationnel. Le rapport d'évaluation regroupe les commentaires et les recommandations 
des traducteurs. Ce premier contact permet au Bureau de faire des constatations intéressantes dont voici les grandes lignes.

Sur le plan ergonomique, le Bureau remarque d'abord une nette différence dans l'expérience vécue par les traducteurs des différents services. Alors qu'une équipe dispose de suffisamment de temps pour se familiariser avec les diverses fonctionnalités du nouvel outil, une autre doit souvent se limiter au traitement de texte en raison des impératifs du service, c'est-à-dire des urgences. Dans un deuxième temps, il se rend compte que l'intégration d'un poste de travail perfectionné entraîne de nombreuses difficultés techniques qui perturbent le travail des traducteurs. Il convient toutefois de souligner que, dans bien des cas, le manque de connaissances préalables du micro-ordinateur de la part du traducteur est à la source des problèmes. En effet, rares sont les traducteurs qui possèdent les connaissances voulues en informatique pour assurer le dépannage.

C'est au niveau de la formation et du temps consacré à l'apprentissage de l'outil que le Bureau tire toutefois la plus grande leçon. Premièrement, avant de procéder à la formation en tant que telle, il est essentiel que les traducteurs maîtrisent très bien le traitement de texte et qu'ils possèdent de bonnes connaissances de base du micro-ordinateur. Il faut ensuite adopter une formule individualisée comportant des exercices pratiques. Toute méthode de formation globale doit être remplacée par une méthode modulaire. On sousestime souvent, au détriment du traducteur, la complexité de l'apprentissage à réaliser.

L'aménagement ergonomique de l'aire de travail revêt également une grande importance. Il est essentiel de prévoir suffisamment d'espace de bureau pour permettre un aménagement fonctionnel. L'encombrement des bureaux force souvent les traducteurs à mettre au point des solutions artisanales aux problèmes d'espace, comme un système de corde et de pinces à linge pour atteindre les divers formats de papier continu derrière les imprimantes.

En ce qui concerne la fonctionnalité du poste, les traducteurs n'aiment pas l'écran pleine page à cause de la petite dimension des caractères en format de 66 lignes. De plus, l'imprimante matricielle est inacceptable en raison des fréquents blocages et du bruit dans un environnement de travail à aires ouvertes. Dans l'ensemble, les traducteurs sont satisfaits des diverses aides du poste. Leurs préférences vont toutefois aux outils de vérification orthographique et grammaticale et au logiciel de gestion de données terminologiques Termex. Pour ce qui est de l'intégrateur, dont l'utilisation peut sembler complexe, le Bureau constate que le degré de satisfaction est largement tributaire du nombre de logiciels utilisés. En effet, un traducteur qui ne maîtrise que le traitement de texte a forcément de la difficulté à intégrer l'aspect multidimensionnel du PTT à ses pratiques de travail. D'autres outils sont jugés inutiles par les traducteurs, tels les logiciels de recherche documentaire et de conversion de fichiers de traitement de texte.

Quant au facteur socio-organisationnel, le Bureau remarque d'emblée une baisse de productivité attribuable au processus d'apprentissage. Il se voit donc obligé de modifier la charge de travail des traducteurs sur PTT et de réduire leurs objectifs de production pendant la première phase du projet pilote.

Compte tenu des souhaits et des recommandations exprimés par les traducteurs, le Bureau décide, au début de 1991, de perfectionner davantage le poste existant en attendant la mise en place de la version réseau (PTT 2), prévue l'année suivante. Cette nouvelle version provisoire, appelée PTT 1.1, présente des améliorations principalement en ce qui a trait à l'ergonomie. Le micro 286 est remplacé par un modèle 386 SX plus performant, et l'écran pleine page cède la place à un écran couleur VGA haute résolution. En outre, les traducteurs disposent chacun d'un lecteur de disque compact leur donnant accès direct à TERMIUM sur DOC. Un mobilier ergonomique mieux adapté est installé. De plus, tous les logiciels sont mis à niveau, et les deux outils jugés inutiles (Textsearch et 
Software Bridge) sont supprimés. Des séances de formation supplémentaire sont également organisées à l'intention des utilisateurs.

À l'automne 1991, le CCRIT livre la version réseau, soit le PTT 2. Il s'agit fondamentalement du PTT 1.1 relié grâce au réseau POWERLan. Un certain nombre de raisons amènent le Bureau à opter pour la mise en réseau, dont plusieurs sont issues des préoccupations exprimées par les traducteurs. D'abord, un réseau facilite l'archivage, la recherche et l'extraction de versions électroniques de textes déjà traduits. Deuxièmement, il simplifie la mise à jour, l'épuration et la fusion des fichiers électroniques de terminologie. Outre qu'il permet l'accès à des fichiers de données et à des programmes communs, le réseau favorise un partage plus rationnel et plus économique des appareils périphériques tels les imprimantes au laser et les lecteurs de disque compact. Ce type de lecteur permet notamment l'interrogation simultanée, à partir de plusieurs postes de travail, de la version de TERMIUM sur DOC, ainsi que d'autres ressources documentaires précieuses se présentant également sous forme de disque compact.

En associant de près les traducteurs au développement et à l'évaluation du poste de travail, le Bureau compte établir les conditions préalables à une implantation plus large du PTT au sein de ses services. Certaines des composantes du PTT sont évaluées en vue de la mise en service d'une trousse informatique plus modeste destinée à l'ensemble des traducteurs du Bureau. Une fois l'essai opérationnel du PTT 2 terminé, ce dernier pourra être implanté dans des services déterminés où des besoins précis auront été cernés.

Toujours dans une optique de bureautisation, le Bureau lance le projet LATTER (L'ATelier du TERminologue) en juin 1990. Il s'agit de la mise au point d'un poste de travail informatisé répondant aux besoins particuliers des terminologues. Le LATTER doit permettre notamment le dépouillement électronique de documents. Le terminologue pourra créer et gérer des fichiers terminologiques à partir de ce dépouillement et les fusionner, le cas échéant, à la base de terminologie TERMIUM. De plus, il sera en mesure d'exécuter l'avant-traitement des fiches des traducteurs créées à l'aide du logiciel Termex (qui fait partie du PTT) en vue de leur ajout à TERMIUM. Un protocole de rédaction de fiches électroniques devant permettre d'uniformiser les paramètres utilisés par les traducteurs et les terminologues est actuellement en voie de préparation. Le premier prototype LATTER devrait entrer en service en avril 1992.

Le Bureau ne s'est toutefois pas cantonné dans les projets pilotes touchant la technologie de pointe. Il a voulu que tous les traducteurs puissent, certes à des niveaux divers, profiter des retombées des percées technologiques. Pour ce faire, il poursuit, depuis près de trois ans, un programme intensif d'informatisation. C'est ainsi que tous les traducteurs possèdent dorénavant une station de travail constituée d'un micro-ordinateur IBM compatible, du traitement de texte WordPerfect et d'un vérificateur orthographique. De plus, certains logiciels sont mis à la disposition des plus aguerris en informatique, qui désirent disposer d'une plus grande variété d'outils électroniques. Ces stations, plus modestes que le PTT, confèrent néanmoins aux traducteurs une grande autonomie. Le travail du traducteur se transforme au fur et à mesure que les nouvelles technologies font leur apparition, suscitant dans leur sillage de nouveaux besoins et de nouvelles attentes chez les clients.

L'expertise du Bureau de la traduction en technologie langagière englobe maintenant plusieurs champs. Les divers travaux entrepris au fil des ans ont permis la mise au point d'un logiciel de traduction automatique (Météo), la création de l'une des plus grandes banques de terminologie au monde (TERMIUM) ainsi que la conception d'un poste de travail adapté aux besoins des traducteurs (PTT) et d'un poste semblable destiné aux terminologues (LATTER). Viennent s'ajouter à la liste l'essai opérationnel du logiciel Logos et la mise au point d'une méthodologie d'évaluation linguistique et technique de logiciels de traduction et d'outils électroniques. 
L'intégration harmonieuse de tous les outils constituera probablement la prochaine étape. En effet, dans un avenir plus lointain, le Bureau espère pouvoir mettre au point un système ultra-perfectionné regroupant la banque TERMIUM, un logiciel de traduction automatique, un logiciel d'éditique et les meilleurs outils électroniques offerts sur le marché. Ce système intégré, encore plus performant et plus convivial que le PTT, permettra l'accès direct par communication à une foule de bases de données terminologiques et documentaires. Il convient toutefois de souligner que ce projet n'en est qu'à l'état embryonnaire.

Entre-temps, le Bureau désire mettre à profit son expérience en TAO et exploiter ses ressources à pleine capacité. C'est dans ce contexte qu'il a chargé le Secrétariat général TAO d'évaluer l'utilisation des ressources en TAO et d'examiner les domaines d'application afin d'optimiser le système Logos. En 1992, la TAO prendra probablement un nouveau visage tout en continuant de se vouloir le reflet et l'actualisation de nouveaux besoins.

Le Bureau de la traduction poursuit sans relâche ses projets de technologie langagière afin de demeurer présent sur le front de la recherche et du développement dans le domaine. L'intérêt des traducteurs demeure vif à cet égard, et de nouvelles initiatives ne cessent de voir le jour.

\section{BIBLIOGRAPHIE}

BOURBEAU, Laurent (1990) : Élaboration et mise au point d'une méthodologie d'évaluation linguistique de systèmes de traduction assistée par ordinateur, Progiciels Bourbeau Pinard Inc., Hull (Québec).

BOURBEAU, Laurent (1991): Fabrication et mise au point d'un corpus d'évaluation linguistique de systèmes de traduction assistée par ordinateur, Progiciels Bourbeau Pinard Inc., Hull (Québec).

CCRIT ( 1991): Poste de travail pour la traduction, Évaluation de la convivialité, Laval (Québec).

Secrétariat d'État du Canada (1990): Évaluation du logiciel de traduction assistée par ordinateur de Logos, Version 6.0, Hull (Québec). 